\title{
Comparison of the protective effects of sildenafil, vardenafil and tadalafil treatments in ischemia- reperfusion injury in rat ovary
}

\author{
Onder Sakin, Ali Doğukan Anğın, Emine Eda Akalın, Muzaffer Seyhan Cikman, \\ Kayhan Basak, Asuman Orcun Kaptanagasi
}

Kartal Dr Lutfi Kirdar Training and Research Hospital, Kartal, Istanbul, Turkey

\begin{abstract}
Objectives: The aim of this study was to compare the effects of sildenafil, vardenafil and tadalafil in treatment for ischemia/reperfusion injury which is created experimentally in rat ovaries.

Material and methods: For this study, 30 female Wistar albino rats were used, and the rats were separated randomly into five groups consisting of six rats each: normal, torsion-detorsion, torsion-detorsion + sildenafil $1.4 \mathrm{mg} / \mathrm{kg}$, torsion-detorsion + vardenafil $1.7 \mathrm{mg} / \mathrm{kg}$ and torsion-detorsion + tadalafil $5.0 \mathrm{mg} / \mathrm{kg}$. The agents were given intraperitoneally 30 minutes before detorsion. An ovarian torsion procedure was implemented in all other groups for 3 hours with the exception of the normal group. Then, a detorsion procedure was implemented to the groups for 3 hours.

Results: The sildenafil and vardenafil treatments showed protective effect by preventing significant increase in inflammation parameters. $(p=0.058,0.138)$. The tadalafil treatment was only protective for cellular degeneration $(p=0.140)$. The vardenafil treatment was protective for edema $(p=0.238)$, vascular congestion $(p=0.111)$, inflammation $(p=0.138)$ and cellular degeneration $(p=0.532$ ). Sildenafil, vardenafil and tadalafil inhibited the increase of atretic follicle. AMH levels were statistically different between torsion and detorsion and vardenafil group $(p=0.004,0.004)$, whereas tadalafil and sildenafil groups were similar to normal group $(p=0.108,0.108)$.

Conclusions: PDE inhibitors were found to be effective in reducing ovarian ischemia/reperfusion injury. Sildenafil and tadalafil seem to be more effective than the vardenafil in protecting the ovarian reserve.
\end{abstract}

Key words: adnexal torsion; ischemia-reperfusion injury; sildenafil; vardenafil; tadalafil; rat model

Ginekologia Polska 2019; 90, 9: 513-519

\section{INTRODUCTION}

Ovarian torsion refers to a complete or partial rotation of the infundibulopelvic or utero ovarian ligament, resulting in ischemic changes in the ovary. Ovarian torsion accounts for $2.7 \%$ of all the gynecologic emergencies [1]. Ovarian torsion could occur at all ages but it is more common in reproductive ages, especially early $20 \mathrm{~s}$ and mid $30 \mathrm{~s}$ [2].

Early diagnosis and treatment is essential for protecting ovarian injury and fertility [3]. In case of delayed diagnosis and intervention, it may cause to impairment or loss of fertility [4]. When ovarian damage occurs; follicular reserves decrease, infertility and early menopause risk increases [5]. Ovarian reserves can be assessed by serum markers or follicle counts [6].

This course of ovarian torsion/detorsion is called as ischemia/reperfusion (I/R) damage [7]. Reperfusion of the ischemic tissue may cause more serious damage to the tissue than ischemia-induced damage [8]. Reperfusion exacerbates ischemic injury at cellular level through reactive oxygen radicals [9]. The release of inflammatory mediators and free oxygen radicals from plasma, leukocytes and vascular cells results in response to inflammatory agents [10].

Nitric oxide (NO), a powerful vasodilator, is an important source of free radical production in I/R injury and NO levels decreases in case of endothelial dysfunction. Neutrophil and platelet adhesion, collection and activation are limited to NO. NO also inhibits lipid peroxidation chain reactions via second precursor cyclic guanosine monophosphate (cGMP) that is cleaved by the phosphodiesterase (PDE) enzyme [11, 12].

The PDE-5 enzyme regulates CGMP catabolism in vascular beds. Sildenafil has been shown to increase the effect of NO by increasing cellular cGMP levels. Increase in cGMP 
levels provides smooth muscle relaxation and an increase in tissue blood flow [13].

Due to all these effects, phosphodiesterase inhibitors have been used widely in many areas. In the literature, sildenafil, vardenafil and tadalafil have been shown to be effective in prevention of I/R damage in many different tissues. Sildenafil has protective effects on $\mathrm{I} / \mathrm{R}$ damage in tissues such as heart, liver, lung, kidney, colon and testis [14-18]. Vardenafil has also been reported to be effective in the treatment of liver, brain and ovarian injuries in $\mathrm{l} / \mathrm{R}$ injury [19].

There are a few recent trials published that investigated the effectiveness of PDE inhibitors in the prevention/treatment of ovarian I/R damage and these trials suggested that PDE inhibitors are effective at these kinds of injuries. However, there are no studies investigating the effects of these drugs on ovarian follicles and their effects on AMH levels. In addition, there is no research evaluating the differences between efficacy and success among these agents.

Our aim in this study is to evaluate the success of PDE inhibitors in ovarian I/R injury and to compare the differences between agents.

\section{MATERIAL AND METHODS}

This study was conducted at the Animal Testing Laboratory of Marmara University after the approval of the Ethics Committee (dated on November 5, 2018; protocol No. 102.2018.mar)

\section{Laboratory animals and the care of animals in research}

Ten-twelve weeks old, female Wistar Albino (Rattus Norvegicus species) rats weighting 200 to 250 grams were used in this study. Rats received light exposure 12 hours a day (from 08:00 to 20:00) and had access to food (standard rodent pellet) and drinking water (tap water) without restriction and kept at room temperature of 21 to $23^{\circ} \mathrm{C}$ and a humidity of 40 to $50 \%$ and were housed 4 or 5 per cage. The number of rats was chosen in in the light of previous studies. Rats were randomly assigned to four groups of 6 . Considering bowel transit time, rats were not fed within 6 hours before laparotomy to empty the gut and allow surgery, but they had access to drinking water.

\section{Groups}

For this study, 30 female Wistar albino rats were used, and the rats were separated randomly into five groups consisting of six rats each: normal, torsion-detorsion, torsion-detorsion + sildenafil $1.4 \mathrm{mg} / \mathrm{kg}$, torsion-detorsion + vardenafil $1.7 \mathrm{mg} / \mathrm{kg}$ and torsion-detorsion + tadalafil $5.0 \mathrm{mg} / \mathrm{kg}$.

Group 1 (normal ovary group - Group N): this group of rats underwent laparotomy once. During the laparotomy, one of the ovaries was removed and fixed in $10 \%$ formaldehyde. And at least $1 \mathrm{~mL}$ of blood sample was taken for AMH test.

Group 2 (torsion ovary group - Group O): Laparotomy was performed and one of the ovaries was twisted 720 degrees and untwisted 3 hours later and the surgical wound was closed without administering any medicine. A second surgery was performed 3 hours later and both ovaries were removed. And at least $1 \mathrm{~mL}$ of blood sample was taken for AMH test.

Group 3 (sildenafil group - Group S): At the first laparotomy one of the ovaries was twisted 720 degrees. Sildenafil $1.4 \mathrm{mg} / \mathrm{kg}$ (Degra ${ }^{\circledR}$ film tablet, Deva Ilac, Istanbul, Turkey) was administered intraperitoneally 30 minutes before detorsion. At the second laparotomy, the ovaries were detorsioned and reperfusion was maintained for 3 hours. At the third laparotomy rats were sacrificed and at least $1 \mathrm{~mL}$ of blood was taken for AMH testing and both ovaries were removed by laparotomy.

Group 4 (vardenafil group - Group V): At the first laparotomy one of the ovaries was twisted 720 degrees. Vardenafil $1.7 \mathrm{mg} / \mathrm{kg}$ (Levitra ${ }^{\circledR}$ film tablet, Bayer, Istanbul, Turkey) was administered intraperitoneally 30 minutes before the second laparotomy. At the second laparotomy, the ovaries were detorsioned and reperfusion was maintained for 3 hours. At the third laparotomy rats were sacrificed and at least $1 \mathrm{~mL}$ of blood was taken for AMH testing and both ovaries were removed by laparotomy.

Group 5 (tadalafil group - Group T): At the first laparotomy one of the ovaries was twisted 720 degrees. Tadalafil $5.0 \mathrm{mg} / \mathrm{kg}$ (Cialis ${ }^{\circledast}$ film tablet, Lilly ilac, Istanbul, Turkey) was administered intraperitoneally 30 minutes before the second laparotomy. At the second laparotomy, the ovaries were detorsioned and reperfusion was maintained for 3 hours. At the third laparotomy rats were sacrificed and at least $1 \mathrm{~mL}$ of blood was taken for $\mathrm{AMH}$ testing and both ovaries were removed by laparotomy.

\section{Surgical procedures}

Sterile, powder-free, latex gloves were used during all surgical procedures. Every rat underwent a laparotomy procedure under anesthesia using 10\% ketamine hydrochloride (Ketalar; Eczacıbaşı, Warner Lambert, Istanbul, Turkey) at a dose of $80 \mathrm{mg} / \mathrm{kg}$ and $2 \%$ xylazine hydrochloride (Rompun; Bayer Health Care LCC, Kansas, KS) at a dose of $15 \mathrm{mg} / \mathrm{kg}$. The procedure was performed while rats were lying in supine position. Abdominal area was shaved before the procedure and the surgical site was prepared using $10 \%$ Povidone-iodine solution (Batticon; Adeka Laboratories, Istanbul, Turkey). A $5 \mathrm{~cm}$ median (on the line between the xiphoid process and pubis) incision was made to enter into the abdominal cavity and the right ovary was twisted 720 degrees along 
with tubo-ovarian blood vessels (Fig. 1). The twisted ovary was fixed to the abdominal muscles with $5 / 0$ silk sutures and the abdominal wall (peritoneum, fascia and skin) was closed in two layers using running locking sutures with 2/0 polyglactin 910 , following bleeding control. Each surgical procedure lasted 15 to 20 minutes to protect the drying effect of the room air and the rats were allowed to wake up.

\section{Histopathological examinations}

Surgically excised ovaries were fixed in $10 \%$ formalin. Paraffin blocks were prepared 24 hours after the oophorectomy procedure. Tissue sections of 5 micrometers were taken and follicular activity was assessed in 5 randomly selected samples

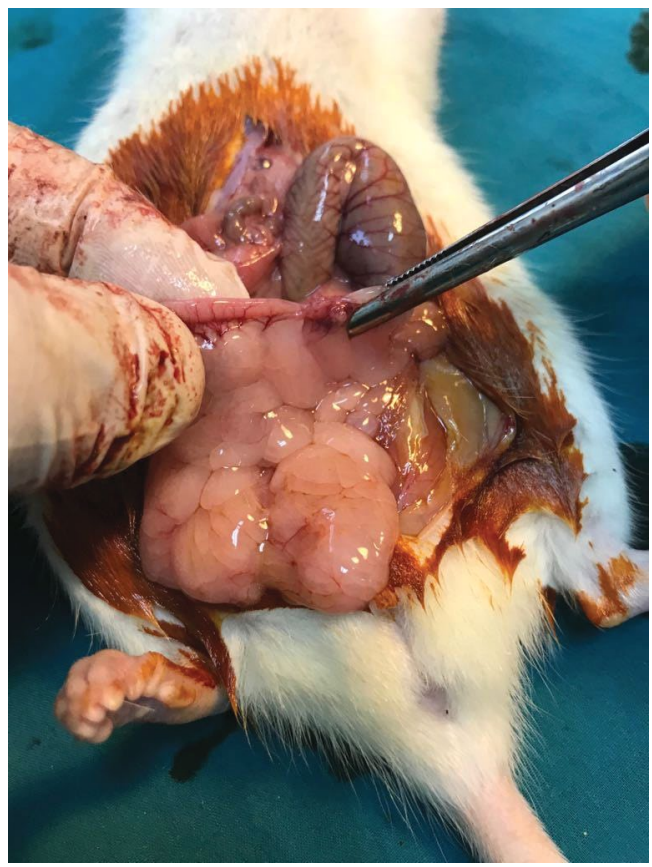

Figure 1. Torsion of ovaries

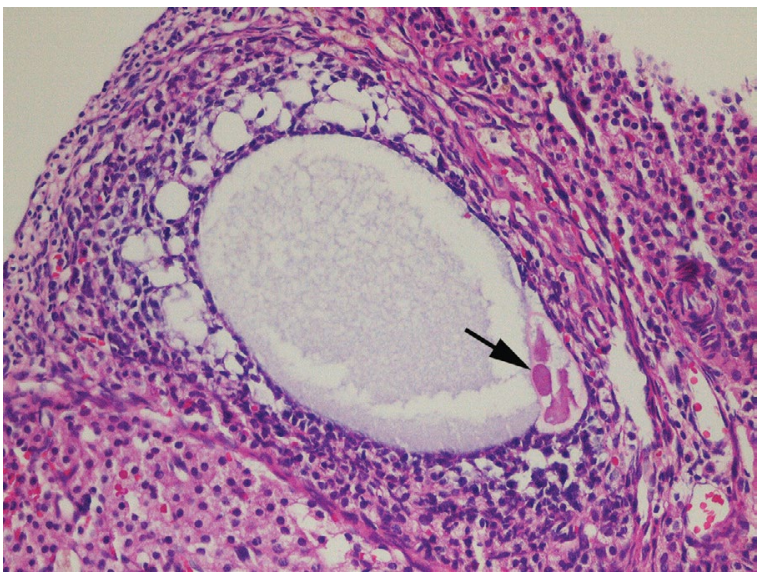

Figure 2. Degenerated oocyte (arrow) in degenerated tertiary follicle; (hematoxylin eosin, $\times 400$ ) from each ovary. Slides were stained with hematoxylin eosin and examined under the light microscope. The paraffin blocks were sectioned using a microtome blade (Leica, Nussloch, Germany). Every slide was blindly assessed by the same pathologist. A light microscope (Olympus Clinical Microscope, Tokyo, Japan) was used to analyze the sections.

Edema, vascular Congestion, inflammation, cellular degeneration and hemorrhage were examined as histopathological injury scores. The scores were evaluated as described by Celik et al. [13]. Pathological findings were rated. Grade 0 indicated normal alterations, no abnormal findings; Grade 1 indicated mild edema, mild vascular congestion, absence of hemorrhage or leukocyte infiltration; Grade 2 indicated moderate edema, moderate vascular congestion, absence of hemorrhage or leukocyte infiltration; Grade 3 indicated severe edema, severe vascular occlusion, minimal hemorrhage and minimal leukocyte infiltration, Grade 4 indicated severe edema, severe vascular occlusion, hemorrhage and leukocyte infiltration (Fig. 2-4).

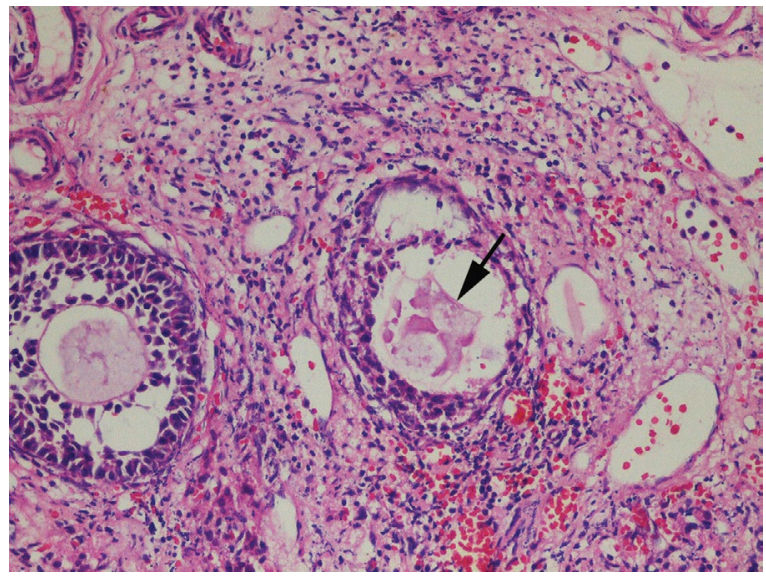

Figure 3. Degenerated secondary follicle and degenerated oocyte in the area of hemorrhage and edema (arrow); (hematoxylin eosin, $\times 400$ )

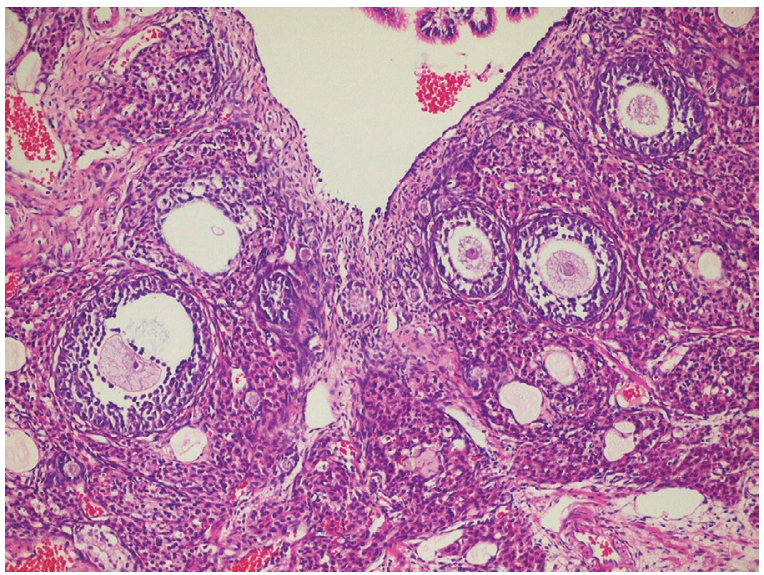

Figure 4. Multiple primordial, primary, secondary and antral follicles in the ovarian cortical region; (hematoxylin eosin, $\times 200$ ) 
All follicles were counted to assess ovarian reserve. Primordial, primary, secondary (pre-antral) and tertiary (antral) follicles were counted.

Follicles were evaluated as described by Parlakgumus et al. [20]. Primordial, primary, secondary (pre-antral) and tertiary (antral) follicles were counted. Primordial follicle is described as an oosit with surrounded only one layer of epithelial cell layer, primer follicle is surrounded with one or more layer of cuboidal granulosa cells. Secondary (pre-antral) follicle is surrounded with more than two cell layers and consists of antrum folliculi and zona pellucida. Tertiary (antral) follicle is defined if there are antrum, stratum granulosum and surrounding cumulus oophorus layers.

\section{Statistical analysis}

Statistical analyses were performed using the SSPS Version 15.0. The Kolmogorov-Smirnov test and histograms were used to assess the normality of the distribution of variables. The mean \pm standard deviation or median (interquartile range) were used to present descriptive analyses. One-way ANOVA test was used to analyze normally distributed numerical data and the Kruskal-Wallis test was used to analyze non-normally distributed numerical data. The limit of statistical significance was set at $p$-values $<0.05$.

\section{RESULTS}

Histopathological damage scores

According to the histopathological damage scores, minimum damage was seen in the normal group and maximum damage was seen in the torsion-detorsion group. There was an increase in all histopathological damage scores (edema, vascular congestion, inflammation, cellular degeneration, hemorrhage) in the torsion group which was not given any drug. (p scores respectively; $<0.001,<0.001,<0.001$, $0.006,<0.001)$.

Hemorrhage were statistically different between normal and torsion group $(p<0.001)$ and PDE inhibitors were not useful for decreasing hemorrhage $(S, V, T$ group; $p=0.002$, $0.002,0.001$ ).

Sildenafil and vardenafil treatments showed protective effect by preventing significant increase in inflammation parameters. ( $p=0.058,0.138$ ) (Tab. 1). Tadalafil treatment was only protective for cellular degeneration $(p=0.140)$. Vardenafil treatment was protective for edema $(p=0.238)$, vascular congestion $(p=0.111)$, inflammation $(p=0.138)$ and cellular degeneration $(p=0.532)$. Vardenafil treatment were effective for $4 / 5$ parameters in histologic examination.

\section{Ovarian follicle counts and AMH levels}

Primiordial, primary, secondary (pre-antral) and tertiary (antral) follicles counts were similar between all the groups. After three hours of ischemia and reperfusion there was not significantly decrease in the study groups than the normal group. Atresic follicle counts and AMH levels were statistically different between normal and the torsion group. Atresic follicles were significantly increased $(p<0.001)$ and AMH levels were significantly decreased in the torsion group $(p=0.004)$. The increase in atresic follicles was prevented in all groups using PDE inhibitors. In the vardenafil group the increase in the number of atresic follicles was prevented

\begin{tabular}{|c|c|c|c|c|c|c|c|c|c|}
\hline & Normal & Detortion & p* & $\begin{array}{l}\text { Detortion + } \\
\text { Sildenafil }\end{array}$ & $\mathbf{p}^{* *}$ & $\begin{array}{l}\text { Detortion + } \\
\text { Vardenafil }\end{array}$ & $\mathbf{P} * * *$ & $\begin{array}{l}\text { Detortion + } \\
\text { Tadalafil }\end{array}$ & $p^{* * * *}$ \\
\hline \multicolumn{10}{|l|}{ Edema } \\
\hline Mean SD & $0.93 \pm 0.69$ & $2.25 \pm 0.79$ & \multirow{2}{*}{$<0.001$} & $2.33 \pm 0.52$ & \multirow{2}{*}{0.003} & $1.50 \pm 1.05$ & \multirow{2}{*}{0.238} & $2.67 \pm 0.52$ & \multirow{2}{*}{0.005} \\
\hline Median- IQR & $1.00(0.00-1.00)$ & $2.00(2.00-3.00)$ & & $2.00(2.00-3.00)$ & & $1.50(1.00-2.00)$ & & $3.00(2.00-3.00)$ & \\
\hline \multicolumn{10}{|c|}{ Vascular congestion } \\
\hline Mean SD & $0.80 \pm 0.85$ & $2.13 \pm 0.95$ & \multirow{2}{*}{$<0.001$} & $2.83 \pm 0.41$ & \multirow{2}{*}{0.002} & $1.83 \pm 0.75$ & \multirow{2}{*}{0.111} & $2.50 \pm 0.84$ & \multirow{2}{*}{0.028} \\
\hline Median & $1.00(0.00-1.00)$ & $2.00(1.00-3.00)$ & & $3.00(3.00-3.00)$ & & $2.00(1.00-2.00)$ & & $3.00(2.00-3.00)$ & \\
\hline \multicolumn{10}{|c|}{ Inflammation } \\
\hline Mean SD & $0.10 \pm 0.31$ & $0.67 \pm 0.56$ & \multirow{2}{*}{$<0.001$} & $0.67 \pm 0.82$ & \multirow{2}{*}{0.058} & $0.33 \pm 0.52$ & \multirow{2}{*}{0.138} & $0.67 \pm 0.52$ & \multirow{2}{*}{0.019} \\
\hline Median & $0.00(0.00-0.00)$ & $1.00(0.00-1.00)$ & & $0.50(0.00-1.00)$ & & $0.00(0.00-1.00)$ & & $1.00(0.00-1.00)$ & \\
\hline \multicolumn{10}{|c|}{ Cellular degeneration } \\
\hline Mean SD & $0.17 \pm 0.38$ & $0.75 \pm 0.94$ & \multirow{2}{*}{0.006} & $0.33 \pm 0.52$ & \multirow{2}{*}{0.138} & $0.33 \pm 0.52$ & \multirow{2}{*}{0.523} & $0.50 \pm 0.84$ & \multirow{2}{*}{0.140} \\
\hline Median & $0.00(0.00-0.00)$ & $0.50(0.00-1.00)$ & & $0.00(0.00-1.00)$ & & $0.00(0.00-1.00)$ & & $0.00(0.00-1.00)$ & \\
\hline \multicolumn{10}{|l|}{ Hemorrhage } \\
\hline Mean SD & $0.13 \pm 0.35$ & $2.46 \pm 0.78$ & \multirow{2}{*}{$<0.001$} & $2.33 \pm 1.03$ & \multirow{2}{*}{0.002} & $2.17 \pm 0.98$ & \multirow{2}{*}{0.002} & $2.83 \pm 0.41$ & \multirow{2}{*}{0.001} \\
\hline Median & $0.00(0.00-0.00)$ & $3.00(2.00-3.00)$ & & $3.00(1.00-3.00)$ & & $2.50(1.00-3.00)$ & & $3.00(3.00-3.00)$ & \\
\hline
\end{tabular}

$p^{*} ; p^{* *} ; p^{* * *} ; p^{* * * *}$ (Mann Whitney U Test) 
$(p=0.461)$, but the decrease in the AMH level could not be prevented $(p=0.004)$. Sildenafil and tadalafil groups were similar with the normal group regarding athretic follicle count ( $p=0.138,0,108$ respectively) and AMH levels $(p=0.140,0.108$ respectively). They prevented the increase in atresic follicle count and the decrease in AMH levels (Tab. 2).

\section{DISCUSSION}

Histopathological examination of the ovary reveals the ischemia/reperfusion injury of the ovary. I/R is associated with edema, vascular congestion, inflammation, cellular degeneration and hemorrhage. I/R injury were studied in the literature and histologic examination score results varied between the trials.. Also several agents were used to prevent I/R injury and their efficiency varied widely in the trials leading to conflicting findings.

Sildenafil was shown to reduce histopathologic injury after two-hour torsion procedure. Sildenafil doses were compared between groups and were not significantly different when applied $0.7 \mathrm{mg} / \mathrm{kg}$ and $1.4 \mathrm{mg} / \mathrm{kg}$. Congestion, hemorrhage and edema were reduced similar to our study. Further they have assessed oxidative stress index [total antioxidant status (TAS), total oxidant status (TOS) and oxidative stress index (OSI)] and showed that these scores were also reduced [21]. But there were no data about the differences of follicle count and AMH levels between groups.
In another study, sildenafil was found to be effective in I/R damage by providing reduction in antioxidant enzymes (superoxide dismutase, glutathione peroxidase), hemorrhage, degenerative cells and atresic follicles. In this study, AMH examinations were not tested [13].

As a result of two studies investigating the effects of vardenafil on ovarian I/R injury; edema, vascular congestion, hemorrhage and the number of atresic follicles were decreased. In these studies, oxidative stress markers were examined. Pre-antral + antral follicle's and AMH examinations were not performed [19, 22]. In our study, vardenafil showed an effective improvement in all histopathological damage scores. Pre-antral + antral follicles remained similar and the increase of atresic follicles was prevented. However, it was observed that $\mathrm{AMH}$ values decreased.

The results of a study investigating the protective effects of tadalafil on I/R injury resulted in a significant reduction in damage scores such as edema, vascular congestion and hemorrhage after 3 hours of ischemia 12 hours of reperfusion and in another group after 24 hours of reperfusion. However, in this study, there is no $\mathrm{AMH}$ examination and follicular examinations [23]. In our study, we determined that tadalafil provided similar reduction in edema, vascular congestion, inflammation and hemorrhage scores.

Although these agents are in the same drug class, their success in treatment of $\mathrm{I} / \mathrm{R}$ damage is different. We think that

Table 2. Comparison of ovarian follicle counts and AMH levels between groups

\begin{tabular}{|c|c|c|c|c|c|c|c|c|c|}
\hline & Normal & Detortion & $\mathbf{p}^{*}$ & $\begin{array}{l}\text { Detortion + } \\
\text { Sildenafil }\end{array}$ & $\mathbf{p}^{* *}$ & $\begin{array}{l}\text { Detortion + } \\
\text { Vardenafil }\end{array}$ & $\mathbf{p}^{* * *}$ & $\begin{array}{l}\text { Detortion + } \\
\text { Tadalafil }\end{array}$ & $\mathbf{p}^{* * * *}$ \\
\hline \multicolumn{10}{|c|}{ Primordial follicle } \\
\hline Mean SD & $6.07 \pm 5.46$ & $3.38 \pm 2.55$ & \multirow{2}{*}{0.153} & $3.17 \pm 1.47$ & \multirow{2}{*}{0.403} & $3.00 \pm 1.67$ & \multirow{2}{*}{0.285} & $1.83 \pm 0.75$ & \multirow{2}{*}{0.930} \\
\hline Median- IQR & $3.50(2.00-11.00)$ & $2.50(2.00-4.00)$ & & $3.00(2.00-3.00)$ & & $2.50(2.00-5.00)$ & & $2.00(1.00-2.00)$ & \\
\hline \multicolumn{10}{|c|}{ Primer follicle } \\
\hline Mean SD & $10.30 \pm 4.99$ & $8.71 \pm 3.18$ & \multirow{2}{*}{0.451} & $9.67 \pm 3.27$ & \multirow{2}{*}{0.104} & $7.50 \pm 2.66$ & \multirow{2}{*}{0.106} & $8.33 \pm 3.83$ & \multirow{2}{*}{0.417} \\
\hline Median & $8.00(7.00-13.00)$ & $8.00(6.00-11.50)$ & & $9.00(7.00-13.00)$ & & $7.50(6.00-8.00)$ & & $10.00(4.00-11.00)$ & \\
\hline \multicolumn{10}{|c|}{ Secondary (pre-antral) follicle } \\
\hline Mean SD & $5.50 \pm 3.18$ & $5.29 \pm 3.11$ & \multirow{2}{*}{0.642} & $3.50 \pm 0 ? ? .84$ & \multirow{2}{*}{0.680} & $3.33 \pm 1.63$ & \multirow{2}{*}{0.466} & $5.17 \pm 2.48$ & \multirow{2}{*}{0.373} \\
\hline Median & $5.00(3.00-7.00)$ & $4.00(3.00-7.50)$ & & $4.00(3.00-4.00)$ & & $3.00(2.00-4.00)$ & & $5.50(3.00-7.00)$ & \\
\hline \multicolumn{10}{|c|}{ Tersier (antral) follicle } \\
\hline Mean SD & $4.80 \pm 2.34$ & $4.79 \pm 2.08$ & \multirow{2}{*}{0.923} & $5.17 \pm 1.94$ & \multirow{2}{*}{0.738} & $5.00 \pm 2.53$ & \multirow{2}{*}{0.371} & $4.33 \pm 2.16$ & \multirow{2}{*}{0.564} \\
\hline Median & $5.00(3.00-7.00)$ & $5.00(3.00-6.50)$ & & $5.00(5.00-6.00)$ & & $4.00(3.00-7.00)$ & & $3.50(3.00-7.00)$ & \\
\hline \multicolumn{10}{|c|}{ Athresic follicle } \\
\hline Mean SD & $0.03 \pm 0.18$ & $0.96 \pm 1.12$ & \multirow{2}{*}{$<0.001$} & $0.33 \pm 0.52$ & \multirow{2}{*}{0.138} & $0.50 \pm 0.84$ & \multirow{2}{*}{0.461} & $0.50 \pm 0.84$ & \multirow{2}{*}{0.140} \\
\hline Median IQR & $0.00(0.00-0.00)$ & $0.50(0.00-2.00)$ & & $0.00(0.00-1.00)$ & & $0.00(0.00-1.00)$ & & $0.00(0.00-1.00)$ & \\
\hline \multicolumn{10}{|l|}{ AMH } \\
\hline Mean SD & $2.641 \pm 0.95$ & $1.372 \pm 0.66$ & \multirow{2}{*}{0.004} & $1.753 \pm 0.79$ & \multirow{2}{*}{0.108} & $1.090 \pm 0.47$ & \multirow{2}{*}{0.004} & $1.801 \pm 0.55$ & \multirow{2}{*}{0.108} \\
\hline Median & $2.591(1.64-3.70)$ & $1.211(0.95-1.66)$ & & $1.596(1.16-2.01)$ & & $1.070(0.64-1.60)$ & & $1.659(1.37-2.30)$ & \\
\hline
\end{tabular}

$\mathrm{p}^{*} ; \mathrm{p}^{* *} ; \mathrm{p}^{* * *} ; \mathrm{p}^{* * * *}$ (Mann Whitney U Test) 
this can be related with the difference in ischemia/reperfusion duration, chemical success and/or half-life of agents.

Tadalafil is effective 36 hours after the dose, its half-life is 17.5 hours [24] and it is about 4.5 hours longer than sildenafil [23].

Vardenafil is more sensitive to PDE-5 than sildenafil and has similar pharmacokinetic parameters [25-27].

In a study conducted by Yeral et al. [6], it was determined that there was no significant difference in the number of pre-antral + antral and total follicles between the groups with and without albumin treatment after 3 hours of ischemia and 7 days of reperfusion.

In these cases, some questions come to mind. Is it important whether different durations are effective in changes in follicle damage? Does short-term ischemia or short-term reperfusion provide a full effect in the creation of follicular damage? As a result of this important study, 2 hours, 4 hours and 16 hours ischemia was performed. Then reperfusion for 28 days was performed. They reported that the number of follicles does not differ between the torsion ovary and contralateral ovary, but this condition cannot guarantee the survival of the ovarian follicles [28].

In our study, no significant difference was observed between the groups in the number of pre-antral + antral follicles after 3 hours of ischemia and 3 hours of reperfusion.

In another study, 3 hours of ischemia and then 7 days of reperfusion decreased ovarian follicles. Atorvastatin has been shown to help with follicular healing but not AMH. They also pointed out that they did not know whether this follicular recovery was permanent and whether the primordial follicles would turn into functional mature follicles [20].

AMH is a dimeric glycoprotein released from granulosa cells of the antral follicles. Therefore, evaluation of the ovarian reserve with $\mathrm{AMH}$ test after iatrogenic events such as pelvic radiotherapy, chemotherapy, uterine artery embolization or ovarian surgery may be helpful in maintaining fertility at an early stage. AMH expression begins in the primordial follicles, whereas the levels of pre-antral and small antral follicles are highest. According to the literature, AMH level is thought to be a better indicator of ovarian reserve compared to age, FSH, LH, E2 and inhibited B levels [29-31].

When follicles show signs of atresia, anti-mullerian hormone (AMH) decreases. Some cells probably show a higher $\mathrm{AMH}$ expression than proliferative capacity and others that reflect differences in steroidogenic activity. In our study, in the vardenafil group even though the increase in the number of atresic follicles was prevented, AMH levels remained low compared to the normal ovarian group. It is seen that the number of atresic follicles and AMH levels are not in direct relationship with ovarian damage scores and pre-antral + antral follicles [32,33].
When the literature is examined, it is seen that there are decreases in the AMH levels after 3 or 6 hours of ovarian torsion followed by 3 hours, 24 hours or 7 days of reperfusion. It is seen that short-term or long-term I/R events affect AMH levels [20, 31, 34, 35].

As a result of our study, 4/5 of the histopathological damage scores improved with vardenafil treatment, there was no decrease in pre-antral + antral follicles, undesired increase in atresic follicles was prevented but the decrease of $\mathrm{AMH}$ value could not be prevented.

Sildenafil and tadalafil showed partial improvement in histopathological damage scores, no decrease in pre-antral + antral follicles, increased atresic follicles, and $\mathrm{AMH}$ values remained within normal ranges.

\section{CONCLUSIONS}

PDE inhibitors were found to be effective in reducing ovarian ischemia/reperfusion injury. The sildenafil and tadalafil seem to be more effective than the vardenafil in protecting the ovarian reserve.

\section{Statement of ethics}

This study was conducted at the Animal Testing Laboratory of Marmara University after the approval of the Ethics Committee (dated on November 5, 2018; protocol No. 102.2018.mar).

\section{REFERENCES}

1. Aslan M, Erkanli Senturk G, Akkaya H, et al. The effect of oxytocin and Kisspeptin-10 in ovary and uterus of ischemia-reperfusion injured rats. Taiwan J Obstet Gynecol. 2017; 56(4): 456-462, doi: 10.1016/j. tjog.2016.12.018, indexed in Pubmed: 28805600.

2. Pınar N, Soylu Karapınar O, Özcan O, et al. Protective effects of tempol in an experimental ovarian ischemia-reperfusion injury model in female Wistar albino rats. Can J Physiol Pharmacol. 2017; 95(7): 861-865, doi: 10.1139/cjpp-2016-0309, indexed in Pubmed: 28423286.

3. Huang Ci, Hong MK, Ding DC. A review of ovary torsion. Ci Ji Yi Xue Za Zhi. 2017; 29(3): 143-147, doi: 10.4103/tcmj.tcmj_55_17, indexed in Pubmed: 28974907.

4. Sintim-Damoa A, Majmudar AS, Cohen HL, et al. Pediatric Ovarian Torsion: Spectrum of Imaging Findings. Radiographics. 2017; 37(6): 1892-1908, doi: 10.1148/rg.2017170026, indexed in Pubmed: 29019757.

5. Oktem O, Oktay K. Quantitative assessment of the impact of chemotherapy on ovarian follicle reserve and stromal function. Cancer. 2007; 110(10): 2222-2229, doi: 10.1002/cncr.23071, indexed in Pubmed: 17932880.

6. Yeral I, Sayan CD, Karaca G, et al. What is the protective effect of krill oil on rat ovary against ischemia-reperfusion injury? J Obstet Gynaecol Res. 2019; 45(3): 592-599, doi: 10.1111/jog.13876, indexed in Pubmed: 30484932.

7. Behroozi-LakT, Zarei L, Moloody-Tapeh M, et al. Protective effects of intraperitoneal administration of nimodipine on ischemia-reperfusion injury in ovaries: Histological and biochemical assessments in a rat model. J Pediatr Surg. 2017; 52(4): 602-608, doi: 10.1016/j.jpedsurg.2016.09.067, indexed in Pubmed: 28277298.

8. Nayki C, Nayki U, et al. Keskin Cimen F, The effect of rutin on ovarian ischemia-reperfusion injury in a rat model. Gynecol Endocrinol. 2018; 34(9): 809-814.

9. Tokgoz VY, Sipahi M, Keskin O, et al. Protective effects of vitamin D on ischemia-reperfusion injury of the ovary in a rat model. Iran J Basic Med Sci. 2018; 21 (6):593-599, doi: 10.22038/IJBMS.2018.26914.6581, indexed in Pubmed: 29942449.

10. Kolac UK, Ustuner MC, Tekin N, et al. The Anti-Inflammatory and Antioxidant Effects of Salvia officinalis on Lipopolysaccharide-Induced Inflammation in Rats. J Med Food. 2017; 20(12): 1193-1200, doi: 10.1089/jmf.2017.0035, indexed in Pubmed: 29131698. 
11. Centeno JM, Orti M, Salom JB, et al. Nitric oxide is involved in anoxic preconditioning neuroprotection in rat hippocampal slices. Brain Res. 1999;836(1-2):62-69, doi: 10.1016/s0006-8993(99)01610-8, indexed in Pubmed: 10415405.

12. Kass DA, Takimoto $E$, Nagayama T, et al. Phosphodiesterase regulation of nitric oxide signaling. Cardiovasc Res. 2007; 75(2): 303-314, doi: 10.1016/j.cardiores.2007.02.031, indexed in Pubmed: 17467673.

13. Celik M, Aksoy AN, Aksoy H, et al. Sildenafil reduces ischemia-reperfusion injury in rat ovary: biochemical and histopathological evaluation. Gynecol Obstet Invest. 2014; 78(3): 162-167, doi: 10.1159/000363747, indexed in Pubmed: 24942826.

14. Beheshtian A, Salmasi AH, Payabvash S, et al. Protective effects of sildenafil administration on testicular torsion/detorsion damage in rats. World J Urol. 2008; 26(2): 197-202, doi: 10.1007/s00345-008-0243-6, indexed in Pubmed: 18265987.

15. Inan $M, U z Y H$, Kizilay $G$, et al. Protective effect of sildenafil on liver injury induced by intestinal ischemia/reperfusion. J Pediatr Surg. 2013; 48(8): 1707-1715, doi: 10.1016/j.jpedsurg.2012.12.054, indexed in Pubmed: 23932610.

16. Shih PK, Cheng CM, Li HP, et al. Pretreatment with sildenafil alleviates early lung ischemia-reperfusion injury in a rat model. J Surg Res. 2013; 185(2): e77-e83, doi: 10.1016/j.jss.2013.07.010, indexed in Pubmed: 23953793.

17. Choi DE, Jeong JY, Lim BJ, et al. Pretreatment of sildenafil attenuates ischemia-reperfusion renal injury in rats. Am J Physiol Renal Physiol. 2009; 297(2): F362-F370, doi: 10.1152/ajprenal.90609.2008, indexed in Pubmed: 19474186.

18. Uzun $\mathrm{H}$, Konukoglu $\mathrm{D}$, Nuri MK, et al. The effects of sildenafil citrate on ischemic colonic anastomotic healing in rats: its relationship between nitric oxide and oxidative stress. World J Surg. 2008; 32(9): 2107-2113, doi: 10.1007/s00268-008-9661-2, indexed in Pubmed: 18581167.

19. BAŞ $H, K A R A$ Ö, KARA $M$, et al. Protective effect of vardenafil on ischemia-reperfusion injury in rat ovary. TURKISH JOURNAL OF MEDICAL SCIENCES. 2013; 43: 684-689, doi: 10.3906/sag-1207-108.

20. Parlakgumus HA, Aka Bolat F, Bulgan Kilicdag E, et al. Atorvastatin for ovarian torsion: effects on follicle counts, $\mathrm{AMH}$, and VEGF expression. Eur J Obstet Gynecol Reprod Biol. 2014; 175: 186-190, doi: 10.1016/j. ejogrb.2014.01.017, indexed in Pubmed: 24507756.

21. Incebiyik A, Seker A, Camuzcuoglu $H$, et al. Does sildenafil have protective effects against ovarian ischemia-reperfusion injury in rats? Arch Gynecol Obstet. 2015; 291(6): 1283-1288, doi: 10.1007/s00404-0143554-4, indexed in Pubmed: 25416202.

22. Yurtcu E, Togrul C, Ozyer S, et al. Dose dependent protective effects of vardenafil on ischemia-reperfusion injury with biochemical and histopathologic evaluation in rat ovary. J Pediatr Surg. 2015; 50(7): 1205-1209, doi: 10.1016/j.jpedsurg.2014.12.013, indexed in Pubmed: 25783344.

23. Arikan DC, Bakan V, Kurutas EB, et al. Protective effect of tadalafil on ischemia/reperfusion injury of rat ovary. J Pediatr Surg. 2010; 45(11): 2203-2209, doi: 10.1016/j.jpedsurg.2010.07.011, indexed in Pubmed: 21034945.
24. Porst $\mathrm{H}$, Padma-Nathan $\mathrm{H}$, Giuliano $\mathrm{F}$, et al. Efficacy of tadalafil for the treatment of erectile dysfunction at 24 and 36 hours after dosing: a randomized controlled trial. Urology. 2003; 62(1): 121-125, doi: 10.1016/s0090-4295(03)00359-5.

25. Hopps CV, Mulhall JP. Novel agents for sexual dysfunction. BJU Int. 2003; 92(6): 534-538, doi: 10.1046/j.1464-410x.2003.04425.x, indexed in Pubmed: 14511028.

26. Daugan A, Grondin P, Ruault $C$, et al. The discovery of tadalafil: a novel and highly selective PDE5 inhibitor. 2: 2,3,6,7,12,12a-hexahydropyrazino[1,2':1,6]pyrido[3,4-b] indole-1,4-dione analogues. J Med Chem. 2003; 46(21): 4533-4542, doi: 10.1021/jm0300577, indexed in Pubmed: 14521415.

27. Mehrotra N, Gupta M, Kovar A, et al. The role of pharmacokinetics and pharmacodynamics in phosphodiesterase- 5 inhibitor therapy. Int J Impot Res. 2007; 19(3): 253-264, doi: 10.1038/sj.ijir.3901522, indexed in Pubmed: 16988721.

28. Calis P, Bozdag G, Karakoc Sokmensuer L, et al. Does ischemia-reperfusion injury affect ovarian reserve and follicle viability in a rat model with adnexal torsion? Eur J Obstet Gynecol Reprod Biol. 2015; 185: 126-130, doi: 10.1016/j.ejogrb.2014.12.006, indexed in Pubmed: 25575140.

29. Dewailly $D$, Andersen $C Y$, Balen $A$, et al. The physiology and clinical utility of anti-Mullerian hormone in women. Hum Reprod Update. 2014; 20(3): 370-385, doi: 10.1093/humupd/dmt062, indexed in Pubmed: 24430863.

30. Riggs RM, Duran EH, Baker MW, et al. Assessment of ovarian reserve with anti-Müllerian hormone: a comparison of the predictive value of anti-Müllerian hormone, follicle-stimulating hormone, inhibin $\mathrm{B}$, and age. Am J Obstet Gynecol. 2008; 199(2): 202.e1-202.e8, doi: 10.1016/j. ajog.2008.05.004, indexed in Pubmed: 18674663.

31. Kaya $\mathrm{C}$, Turgut $\mathrm{H}$, Cengiz $\mathrm{H}$, et al. Effect of detorsion alone and in combination with enoxaparin therapy on ovarian reserve and serum antimüllerian hormone levels in a rat ovarian torsion model. Fertil Steril. 2014; 102(3): 878-884.e1, doi: 10.1016/j.fertnstert.2014.06.007, indexed in Pubmed: 24996496

32. Visser JA, Durlinger ALL, Peters IJJ, et al. Regulation of ovarian function: the role of anti-Müllerian hormone. Reproduction. 2002; 124(5):601-609, indexed in Pubmed: 12416998.

33. Baarends WM, Uilenbroek JT, Kramer $P$, et al. Anti-müllerian hormone and anti-müllerian hormone type II receptor messenger ribonucleic acid expression in rat ovaries during postnatal development, the estrous cycle, and gonadotropin-induced follicle growth. Endocrinology. 1995; 136(11): 4951-4962, doi: 10.1210/endo.136.11.7588229, indexed in Pubmed: 7588229.

34. Ozler A, Turgut A, Görük NY, et al. Evaluation of the protective effects of $\mathrm{CoQ}_{10}$ on ovarian I/R injury: an experimental study. Gynecol Obstet Invest. 2013; 76(2): 100-106, doi: 10.1159/000353425, indexed in Pubmed: 23886769.

35. Sahin Ersoy G, Eken M, Tal R, et al. N-acetylcysteine leads to greater ovarian protection than enoxaparin sodium in a rat ovarian torsion model. Reprod Biomed Online. 2016; 33(1): 93-101, doi: 10.1016/j. rbmo.2016.03.009, indexed in Pubmed: 27083693. 